\title{
Universal Statistics of Branched Flows
}

\author{
Jakob J. Metzger ${ }^{1,2}$, Ragnar Fleischmann ${ }^{1}$, Theo Geisel ${ }^{1,2}$ \\ ${ }^{1}$ Max-Planck-Institute for Dynamics and Self-Organization, Göttingen, Germany \\ ${ }^{2}$ Department of Physics, University of Göttingen, Germany
}




\section{Universal Statistics of Branched Flows}

Jakob J. Metzger ${ }^{1,2}$, Ragnar Fleischmann ${ }^{1}$, Theo Geisel ${ }^{1,2}$

${ }^{1}$ Max-Planck-Institute for Dynamics and Self-Organization, Göttingen, Germany

${ }^{2}$ Department of Physics, University of Göttingen, Germany

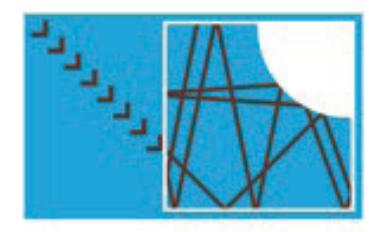

\section{FOR760}
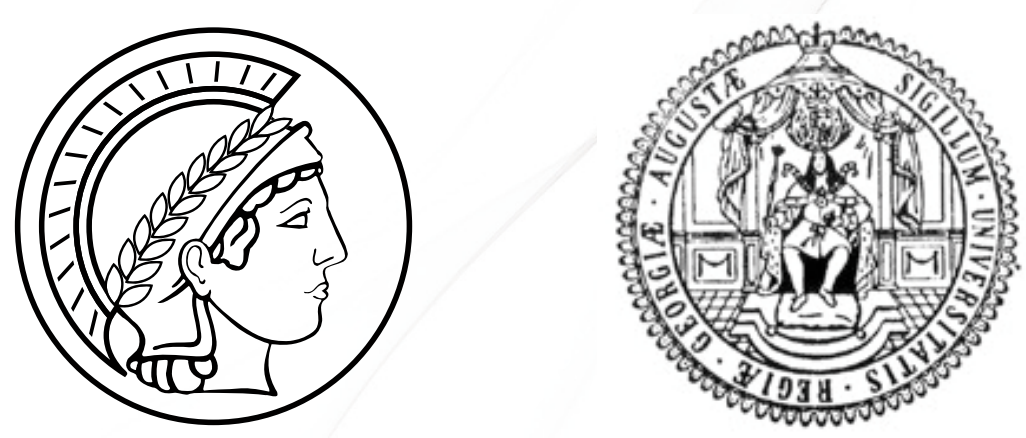

Cargèse, July 2010 


\section{Experimental observation of branched flow}

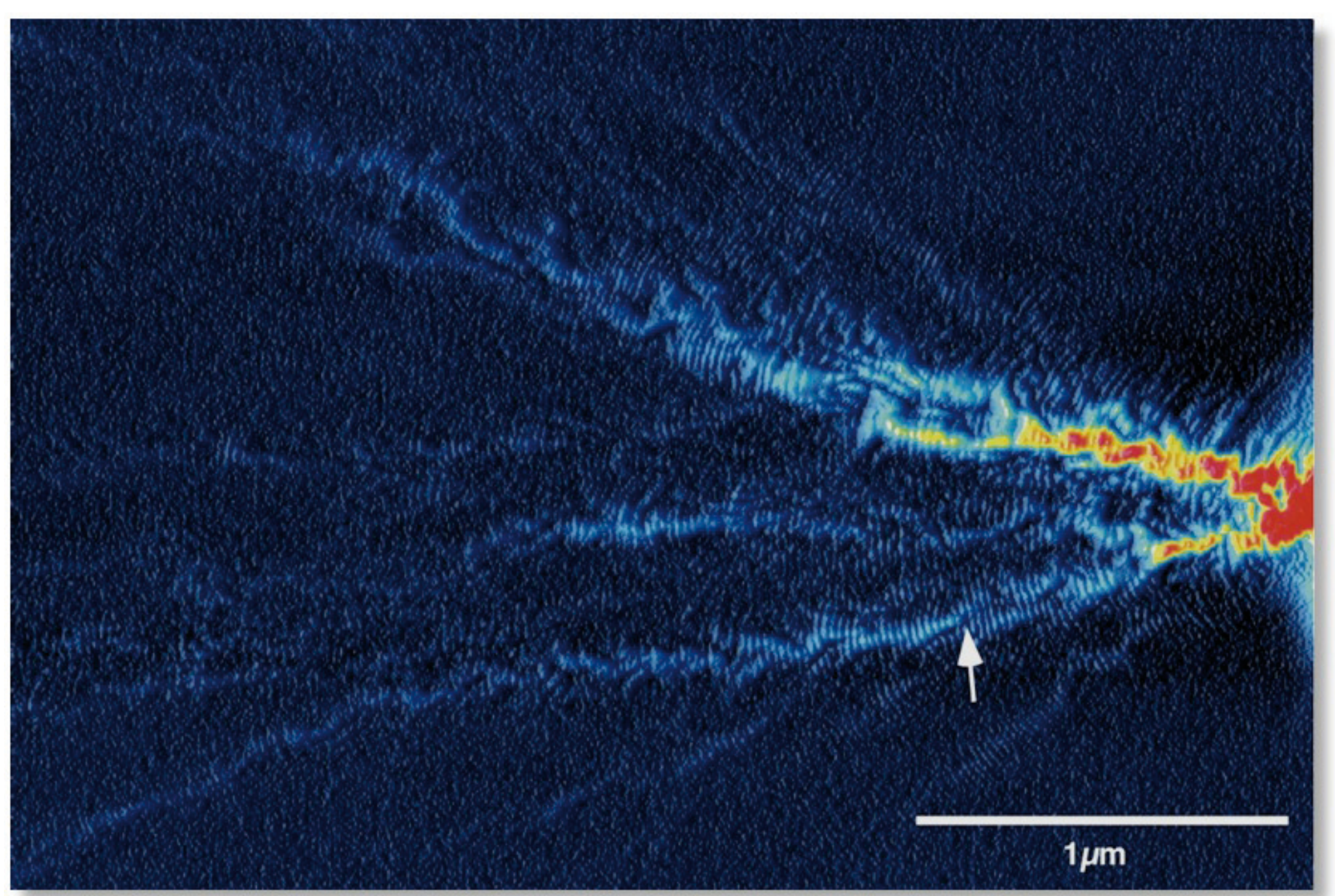

Topinka et al., Nature 410, 183 (2001) 
- Branched flow is a universal phenomenon of 2D Hamiltonian flows in weak, correlated disorder potentials

- Many flows can approximately be described by Hamiltonian flows and suffer from small perturbations by a random potential

- Examples:

- Branched flow in semiconductor devices (with magnetic fields*)

Topinka et al., Nature 410, 183 (2001)

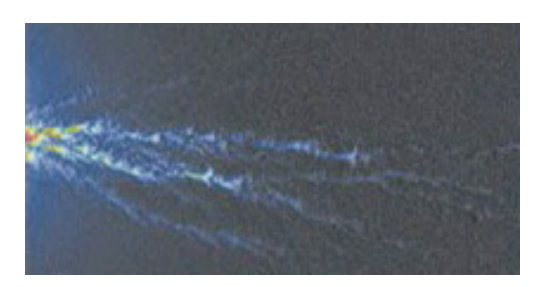

Aidala et al., Nat. Phys. 3 (2007) *

Jura et al., Nat. Phys. 3 (2007)

- Freak waves in microwave cavities Höhmann et al., PRL 104 (2010)

- Rogue sea waves Heller et al., J. Geophys. Res. 113 (2008) Müller et al., Oceanography, 18 (2005)

- Branching of sound waves Wolfson, Tomsovic, J. Acous. Soc. Am. 109 (2001)
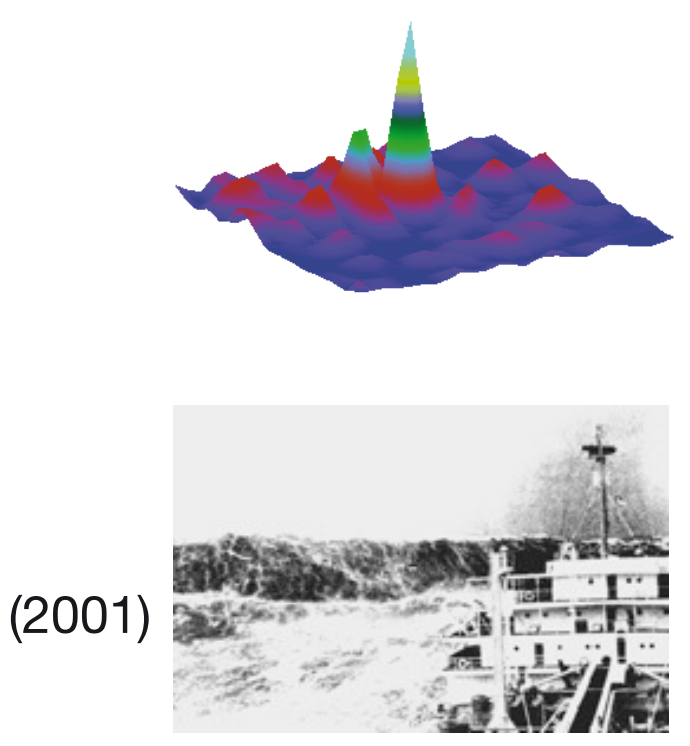
- Motivation

- Intro: Branched Flow and Caustics

- Branch/caustics statistics Approximations and Results

- Conclusion/Outlook 


\section{Caustics and branched flow}

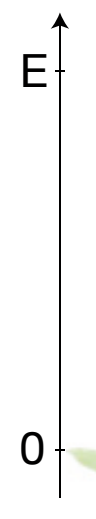

$$
\begin{aligned}
& \left\langle V\left(\mathbf{r}^{\prime}\right) V\left(\mathbf{r}^{\prime}+\mathbf{r}\right)\right\rangle=\epsilon^{2} c\left(|\mathbf{r}| / \ell_{c}\right) \\
& \mathbf{r}=(x, y) \\
& H=\mathbf{p}^{2} / 2+V(\mathbf{r})
\end{aligned}
$$




\section{Caustics and branched flow}
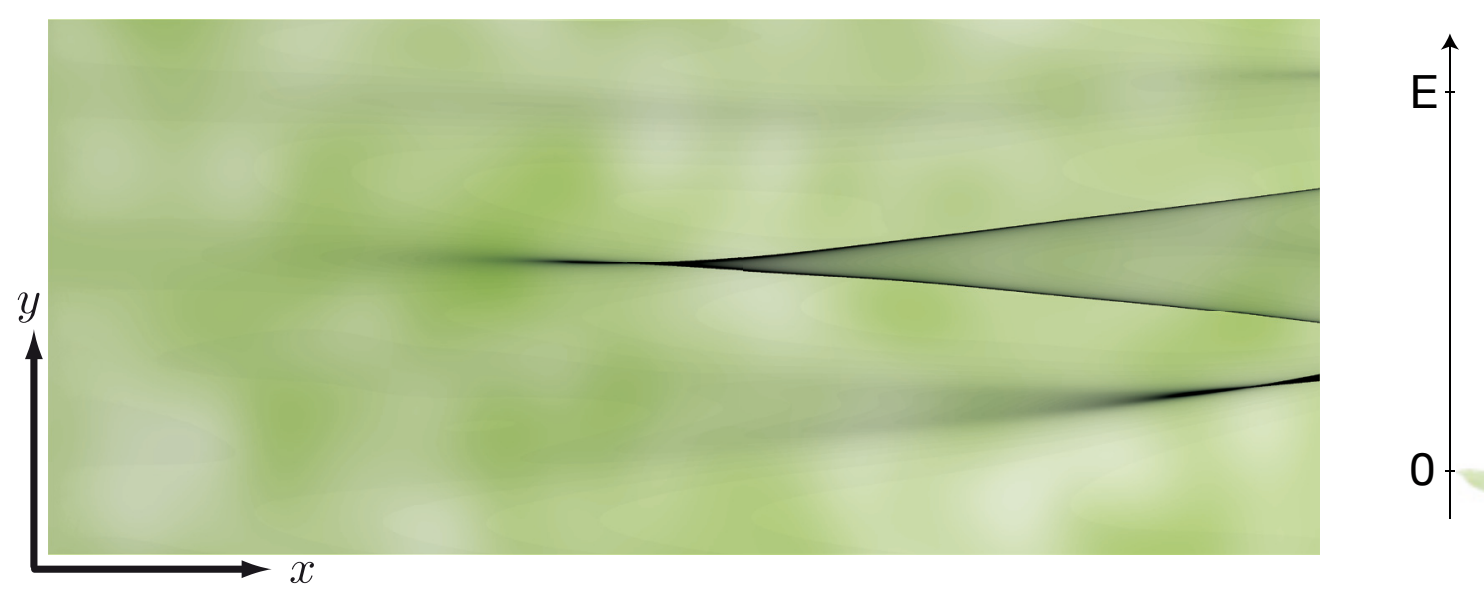

$$
\begin{aligned}
& \left\langle V\left(\mathbf{r}^{\prime}\right) V\left(\mathbf{r}^{\prime}+\mathbf{r}\right)\right\rangle=\epsilon^{2} c\left(|\mathbf{r}| / \ell_{c}\right) \\
& \mathbf{r}=(x, y) \\
& H=\mathbf{p}^{2} / 2+V(\mathbf{r})
\end{aligned}
$$




\section{Caustics and branched flow}
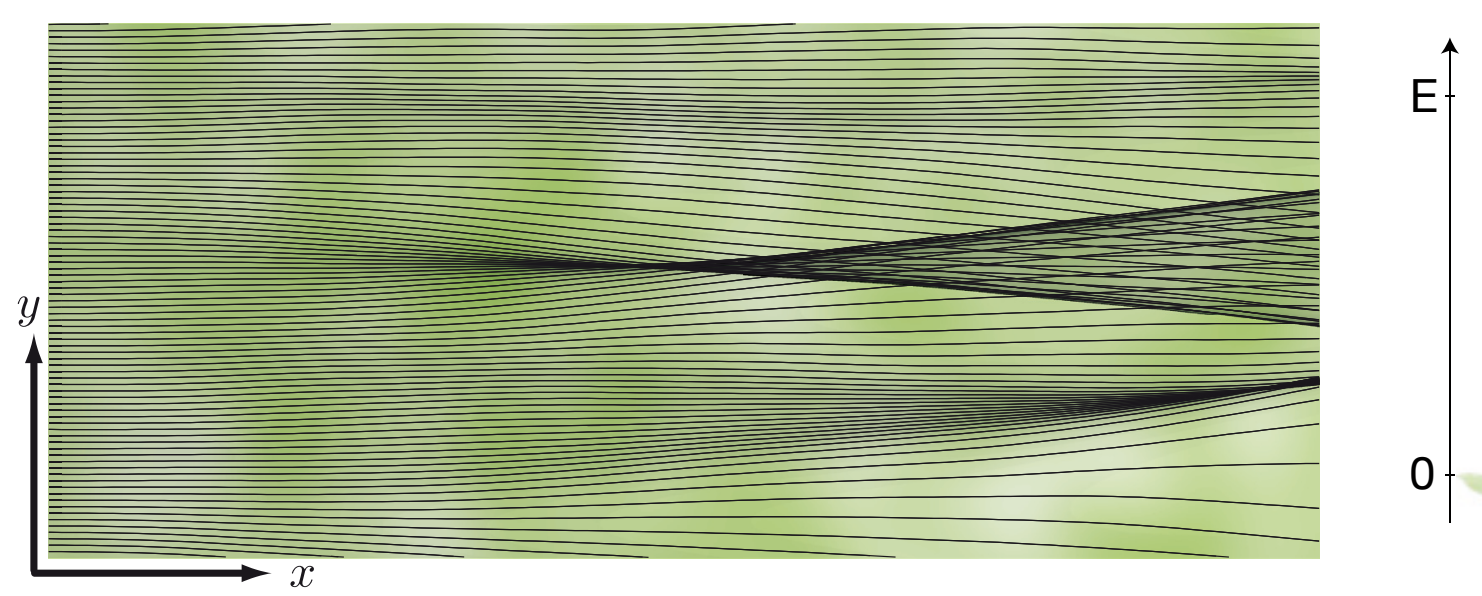

$$
\begin{aligned}
& \left\langle V\left(\mathbf{r}^{\prime}\right) V\left(\mathbf{r}^{\prime}+\mathbf{r}\right)\right\rangle=\epsilon^{2} c\left(|\mathbf{r}| / \ell_{c}\right) \\
& \mathbf{r}=(x, y) \\
& H=\mathbf{p}^{2} / 2+V(\mathbf{r})
\end{aligned}
$$




\section{Caustics and branched flow}
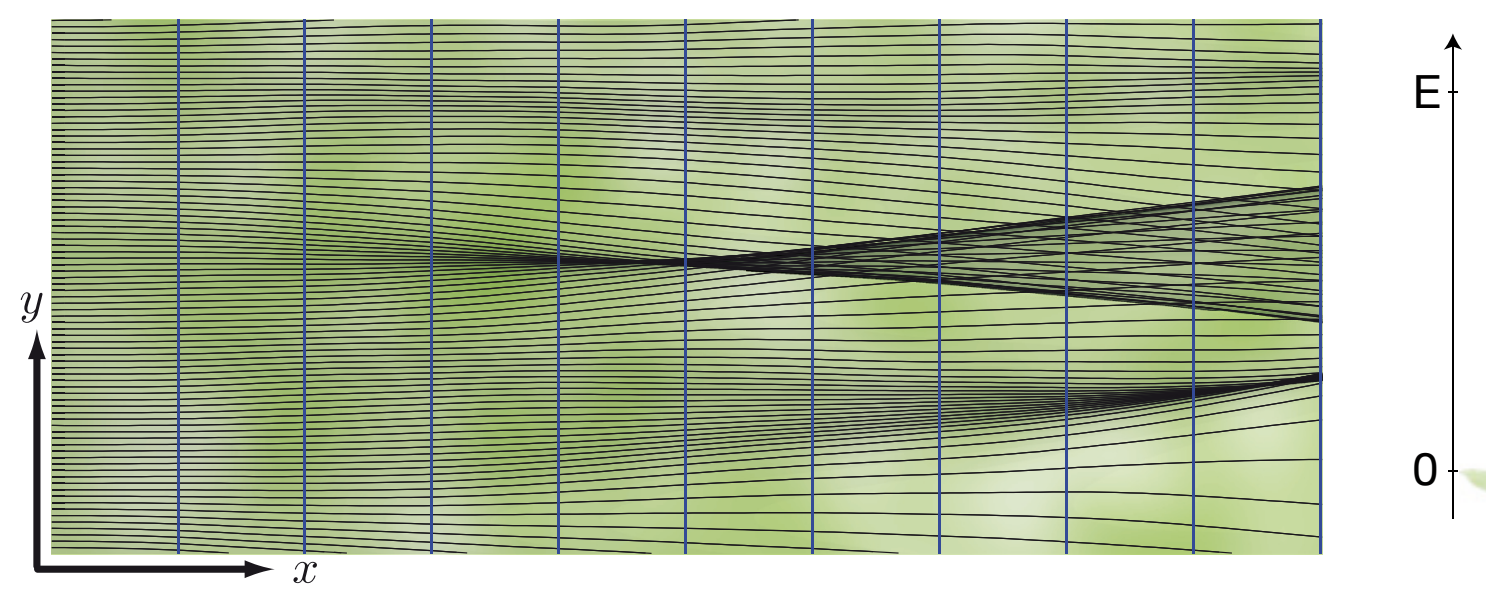

$$
\begin{aligned}
& \left\langle V\left(\mathbf{r}^{\prime}\right) V\left(\mathbf{r}^{\prime}+\mathbf{r}\right)\right\rangle=\epsilon^{2} c\left(|\mathbf{r}| / \ell_{c}\right) \\
& \mathbf{r}=(x, y) \\
& H=\mathbf{p}^{2} / 2+V(\mathbf{r})
\end{aligned}
$$




\section{Caustics and branched flow}
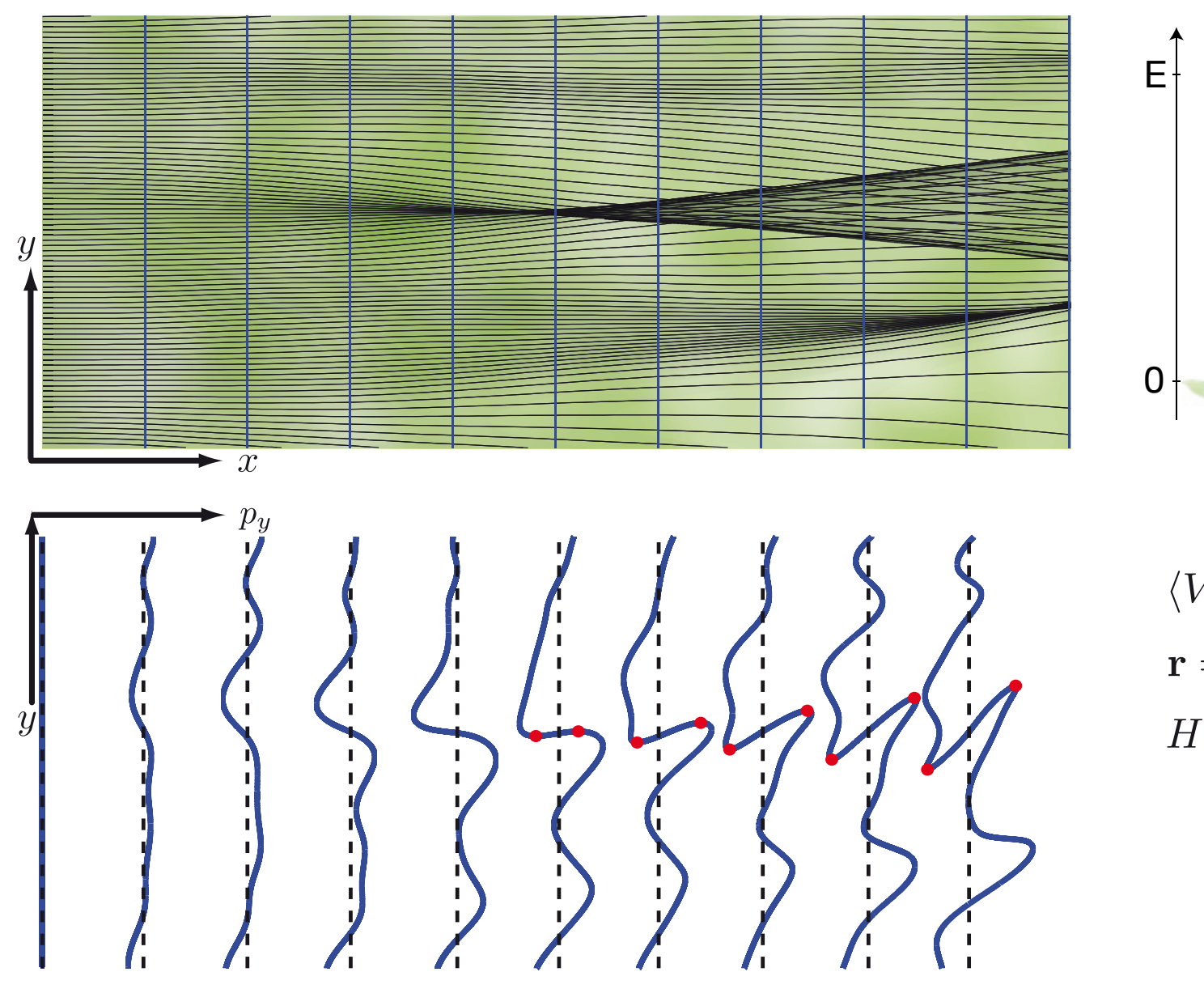

$$
\begin{aligned}
& \left\langle V\left(\mathbf{r}^{\prime}\right) V\left(\mathbf{r}^{\prime}+\mathbf{r}\right)\right\rangle=\epsilon^{2} c\left(|\mathbf{r}| / \ell_{c}\right) \\
& \mathbf{r}=(x, y) \\
& H=\mathbf{p}^{2} / 2+V(\mathbf{r})
\end{aligned}
$$




\section{Caustics and branched flow}
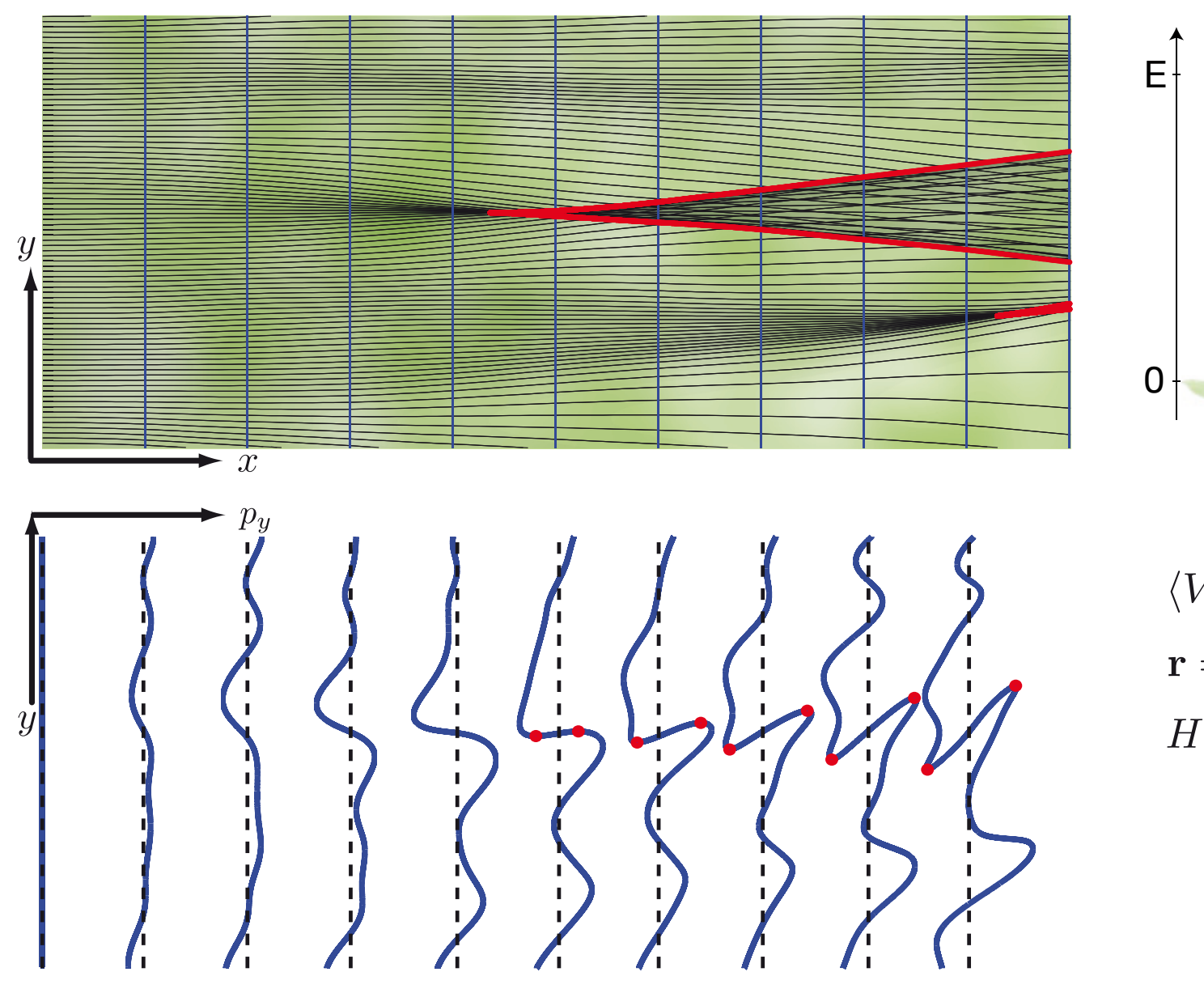

$$
\begin{aligned}
& \left\langle V\left(\mathbf{r}^{\prime}\right) V\left(\mathbf{r}^{\prime}+\mathbf{r}\right)\right\rangle=\epsilon^{2} c\left(|\mathbf{r}| / \ell_{c}\right) \\
& \mathbf{r}=(x, y) \\
& H=\mathbf{p}^{2} / 2+V(\mathbf{r})
\end{aligned}
$$


Caustics and branched flow
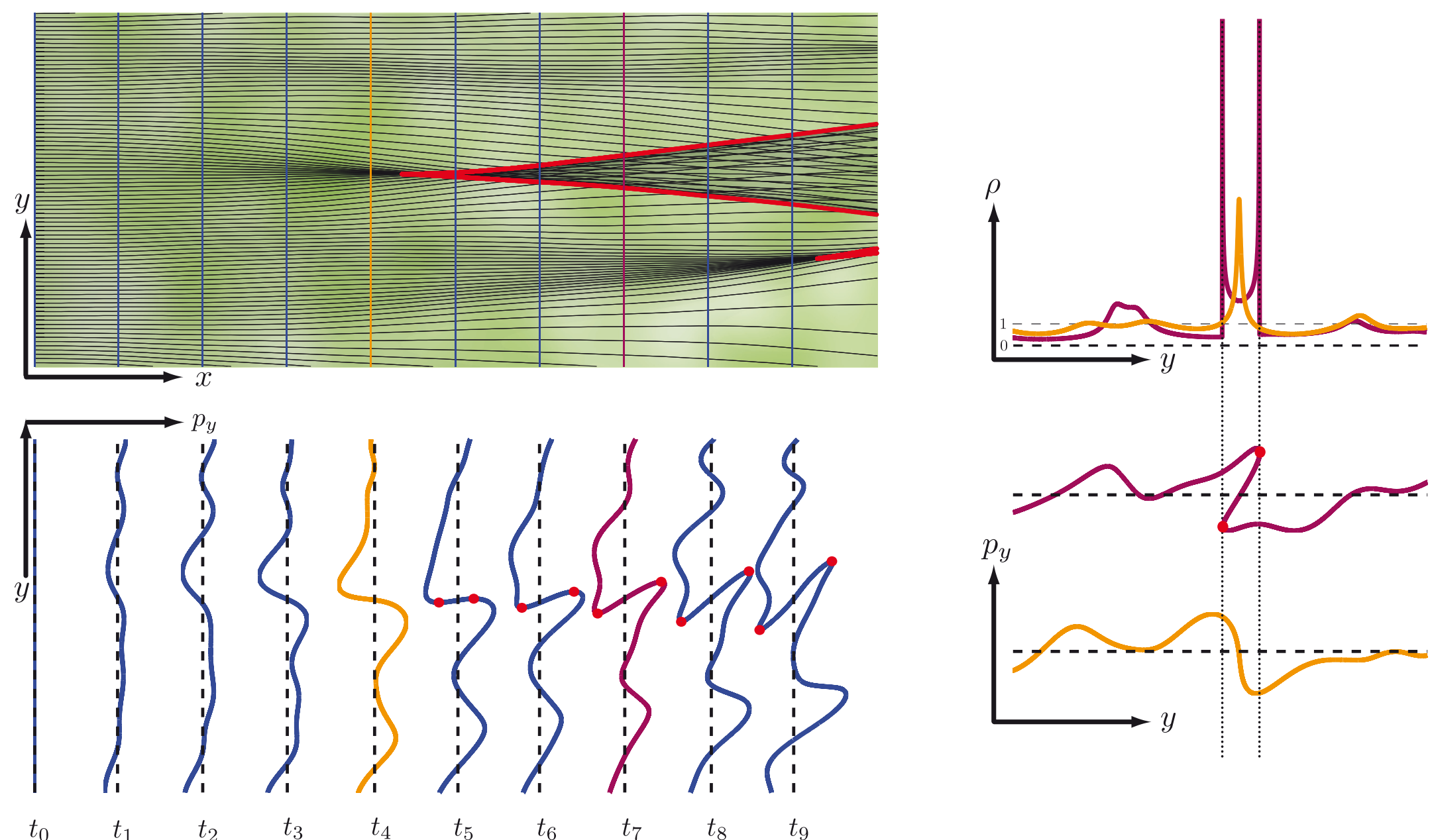
How many branches exist on average per unit length?

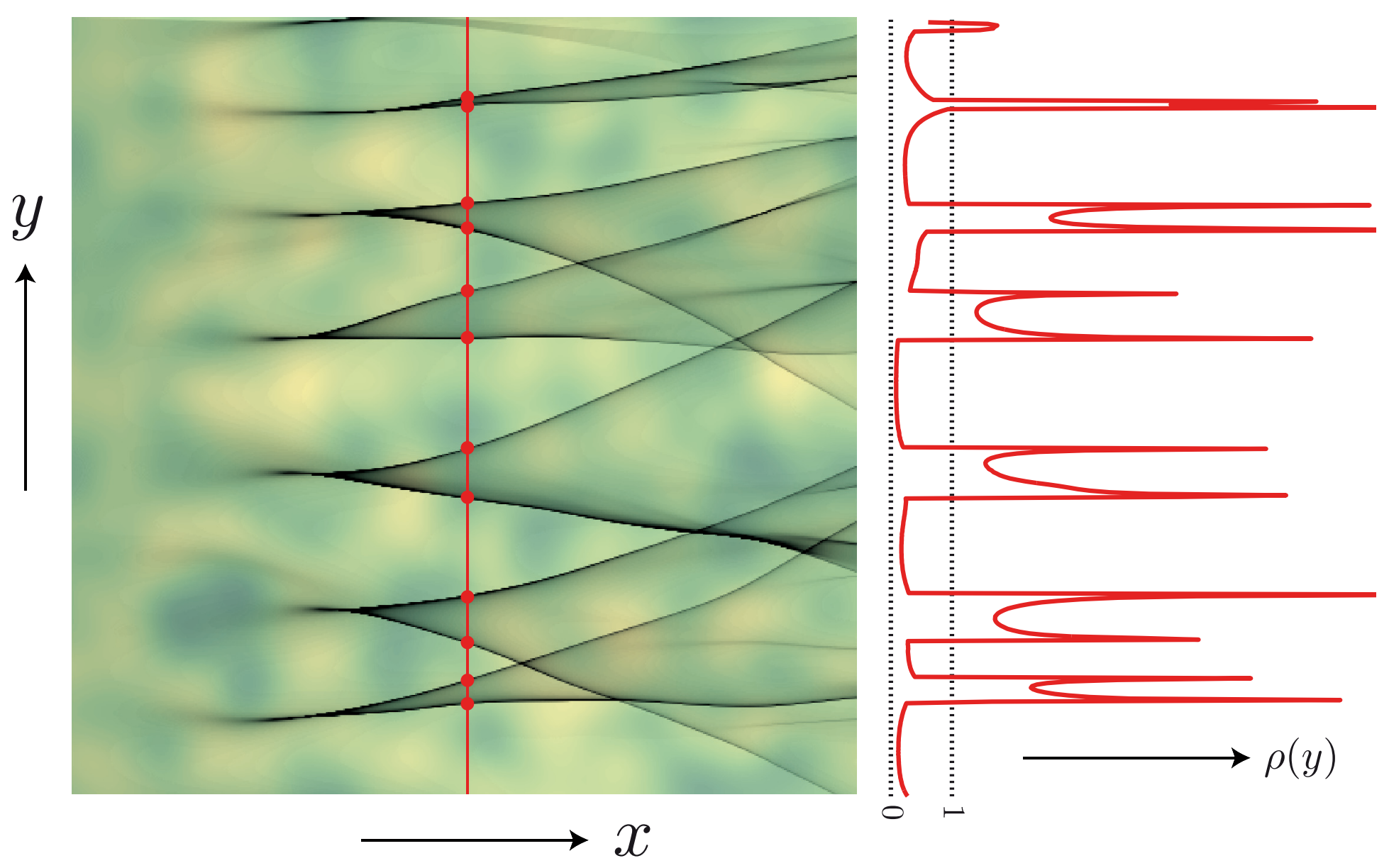


- Kravtsov, Sov. Phys. JETP 28 (1969)

- Berry \& Upstill, Progr. in Optics 18 (1980)

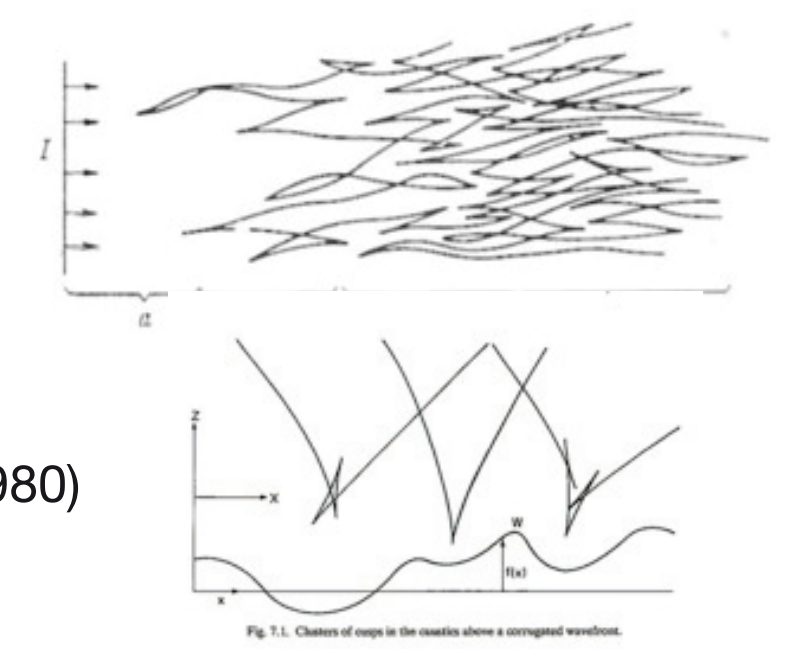

- Kulkarny \& White, Phys. Fluids, 25, (1982)

- White \& Fornberg, J. Fluid Mech. 335 (1998) mean first time to caustic $\left\langle t_{\text {caustic }}\right\rangle \propto \epsilon^{-2 / 3} \ell_{c}$ scaling of mean free path $\quad \ell_{m f p} \propto \epsilon^{-2} \ell_{c}$

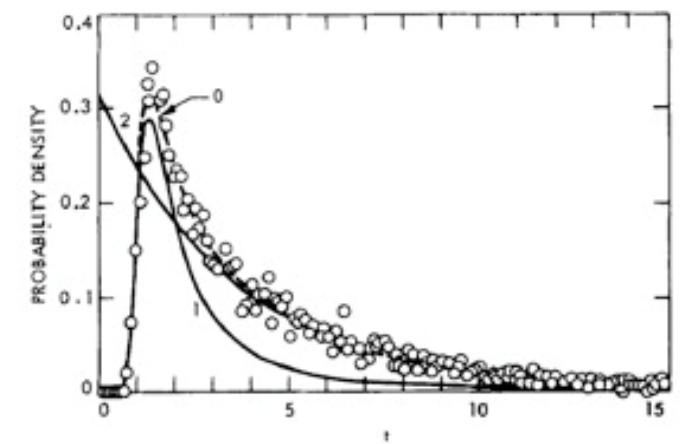

- Kaplan, Phys. Rev. Lett. 89 (2002)

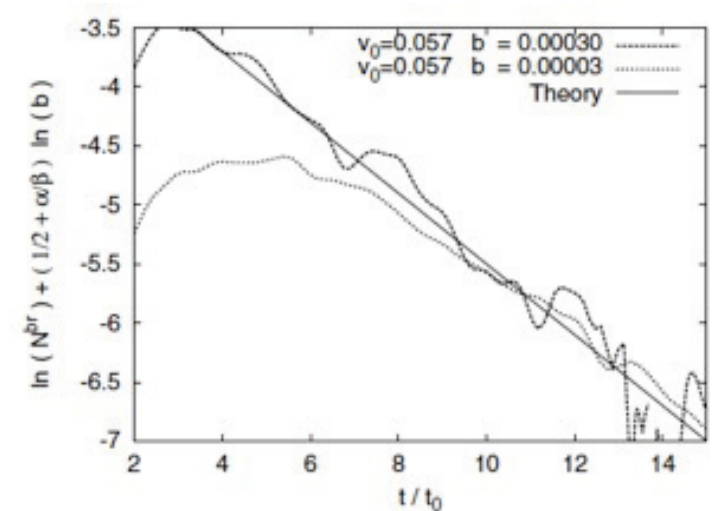




\section{Branch counting}

How many branches transverse to flow exist on average as a function of distance?

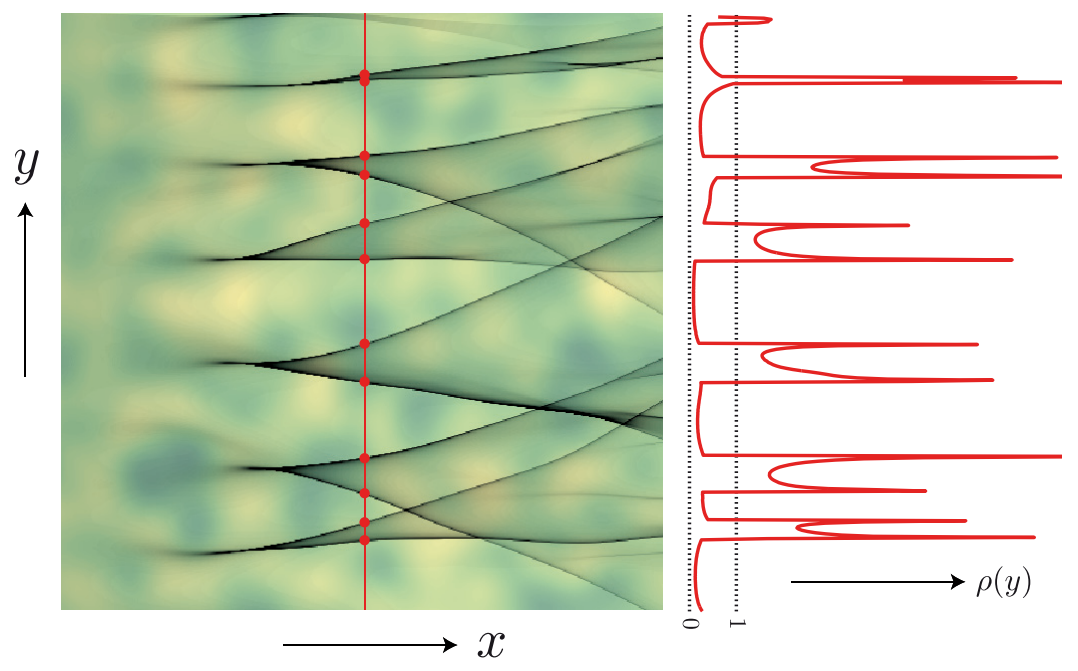

$$
\begin{aligned}
N_{b}(t) & =\frac{1}{2} \lim _{L \rightarrow \infty} \frac{1}{L}\left\langle\int_{0}^{L} d y_{0} \delta\left(m\left(y_{0}, t\right)\right)\left|\partial_{y_{0}} m\left(y_{0}, t\right)\right|\right\rangle \\
& =\frac{1}{2} \lim _{L \rightarrow \infty} \frac{1}{L}\left\langle\int_{0}^{L} d y_{0} \frac{\delta\left(t-t_{c}\right)}{\left|\dot{m}\left(y_{0}, t\right)\right|}\left|\partial_{y_{0}} m\left(y_{0}, t\right)\right|\right\rangle
\end{aligned}
$$

$P_{c}(t) \quad$ probability to caustic along trajectory

[2]

$$
\begin{aligned}
m(t) \equiv \partial y(t) / \partial y(0) & \text { entry of the stability matrix } m_{i j}(t) \equiv \partial \gamma_{i}(t) / \partial \gamma_{j}(0) \\
n(t) \equiv \partial y_{0} m(t) & \text { entry of the extended stability tensor } n_{i j k}(t) \equiv \partial^{2} \gamma_{i}(t) / \partial \gamma_{j}(0) \gamma_{k}(0)
\end{aligned}
$$




\section{Statistics of the stability matrix and tensor}

- Coupled differential equations for entries of the stability matrix and extended stability tensor

- Nonlinear, multiplicative noise

- Obtained closed set of 11 linear differential equations for the moments 
Approximations

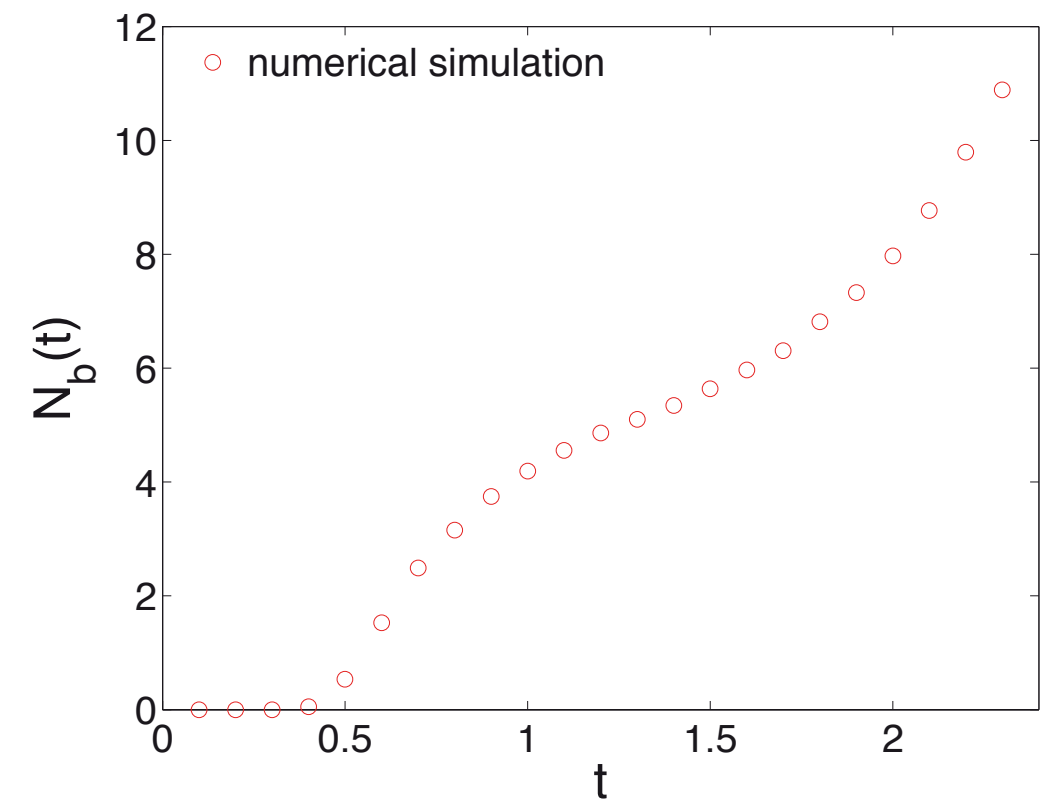




\section{Approximations}

- short-term solution: $N_{b}(t) \approx c_{1}\left(\sigma_{2} / \sigma_{1}\right) t_{0} P_{c}(t) g\left(t / t_{0}\right) \quad t \ll t_{0}$

$$
\begin{aligned}
& \sigma_{1}^{2}=\left.\frac{1}{2} \int_{-\infty}^{\infty} d x \frac{\partial^{4} c(x, y)}{\partial y^{4}}\right|_{y=0} \\
& \sigma_{2}^{2}=-\left.\frac{1}{2} \int_{-\infty}^{\infty} d x \frac{\partial^{6} c(x, y)}{\partial y^{6}}\right|_{y=0}
\end{aligned}
$$

$t_{0} \propto \sigma_{1}$

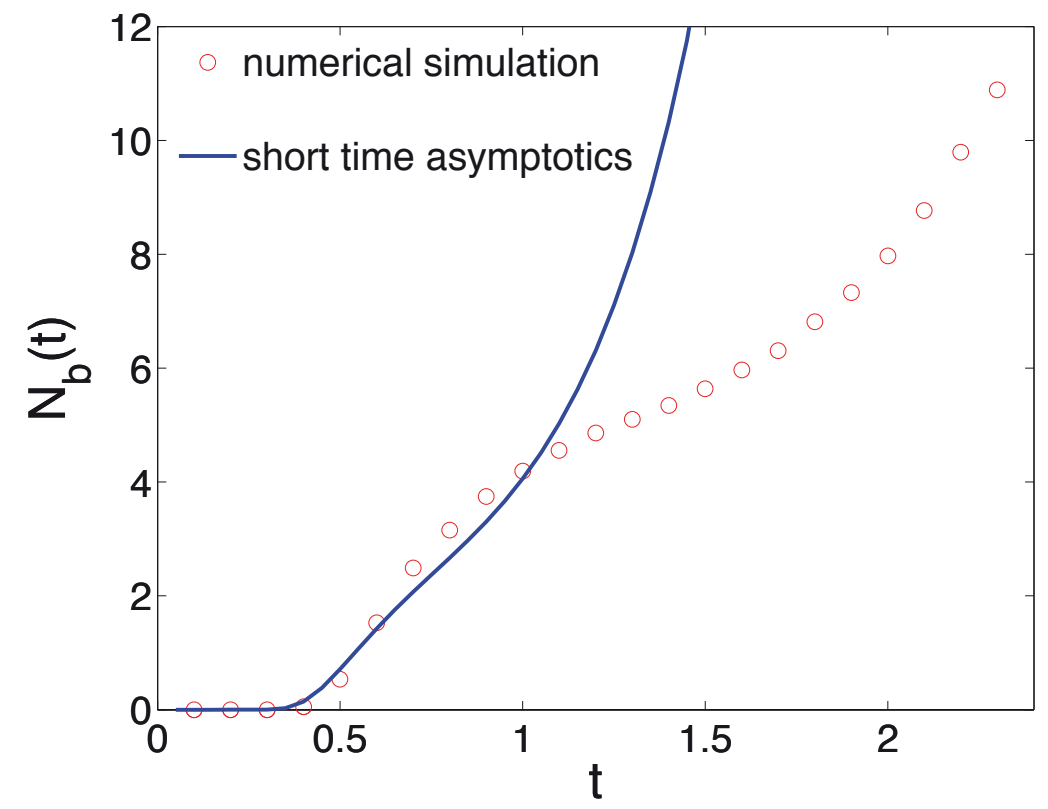




\section{Approximations}

- short-term solution: $N_{b}(t) \approx c_{1}\left(\sigma_{2} / \sigma_{1}\right) t_{0} P_{c}(t) g\left(t / t_{0}\right) \quad t \ll t_{0}$

- long-term solution: $\quad N_{b}(t) \approx c_{2}\left(\sigma_{2} / \sigma_{1}\right) t_{0} P_{c}(t) e^{\lambda\left(t / t_{0}\right)} \quad t \gg t_{0}$

$$
\begin{aligned}
& \sigma_{1}^{2}=\left.\frac{1}{2} \int_{-\infty}^{\infty} d x \frac{\partial^{4} c(x, y)}{\partial y^{4}}\right|_{y=0} \\
& \sigma_{2}^{2}=-\left.\frac{1}{2} \int_{-\infty}^{\infty} d x \frac{\partial^{6} c(x, y)}{\partial y^{6}}\right|_{y=0}
\end{aligned}
$$

$t_{0} \propto \sigma_{1}$

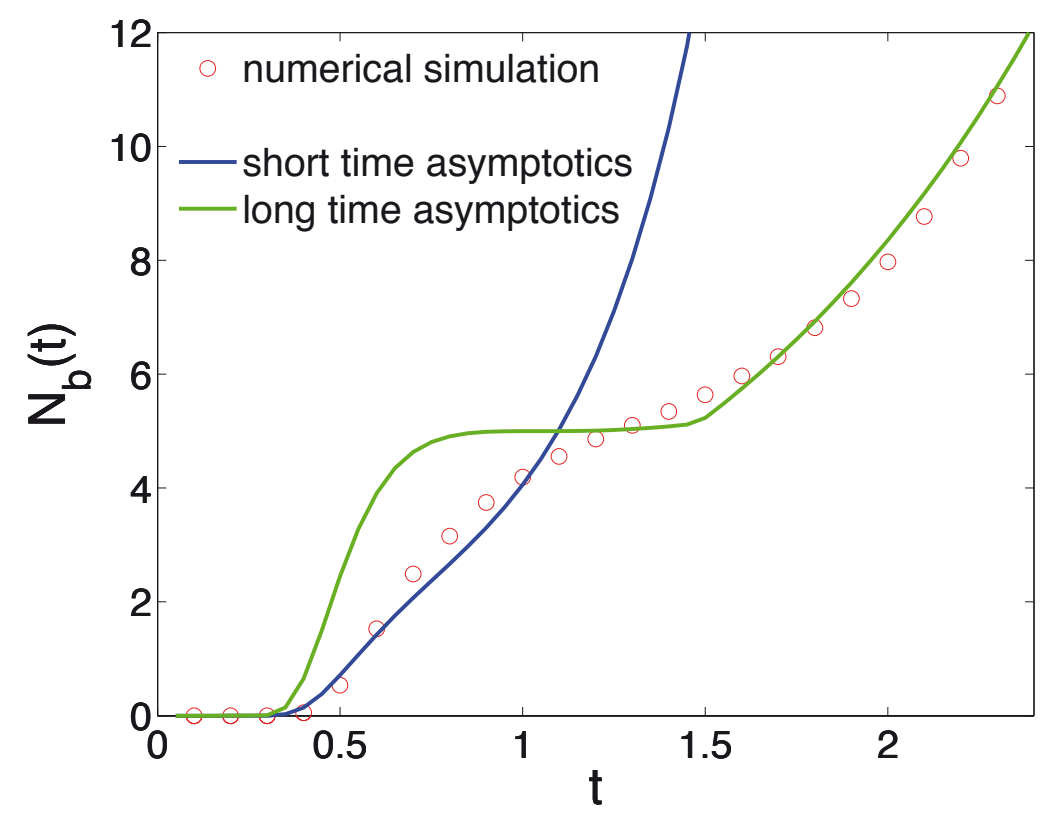




\section{Approximations}

- short-term solution: $N_{b}(t) \approx c_{1}\left(\sigma_{2} / \sigma_{1}\right) t_{0} P_{c}(t) g\left(t / t_{0}\right) \quad t \ll t_{0}$

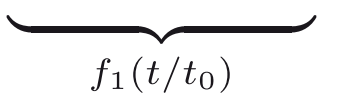

- long-term solution: $N_{b}(t) \approx c_{2}\left(\sigma_{2} / \sigma_{1}\right) t_{0} P_{c}(t) e^{\lambda\left(t / t_{0}\right)} \quad t \gg t_{0}$

$$
\begin{aligned}
& \sigma_{1}^{2}=\left.\frac{1}{2} \int_{-\infty}^{\infty} d x \frac{\partial^{4} c(x, y)}{\partial y^{4}}\right|_{y=0} \\
& \sigma_{2}^{2}=-\left.\frac{1}{2} \int_{-\infty}^{\infty} d x \frac{\partial^{6} c(x, y)}{\partial y^{6}}\right|_{y=0}
\end{aligned}
$$

$t_{0} \propto \sigma_{1}$

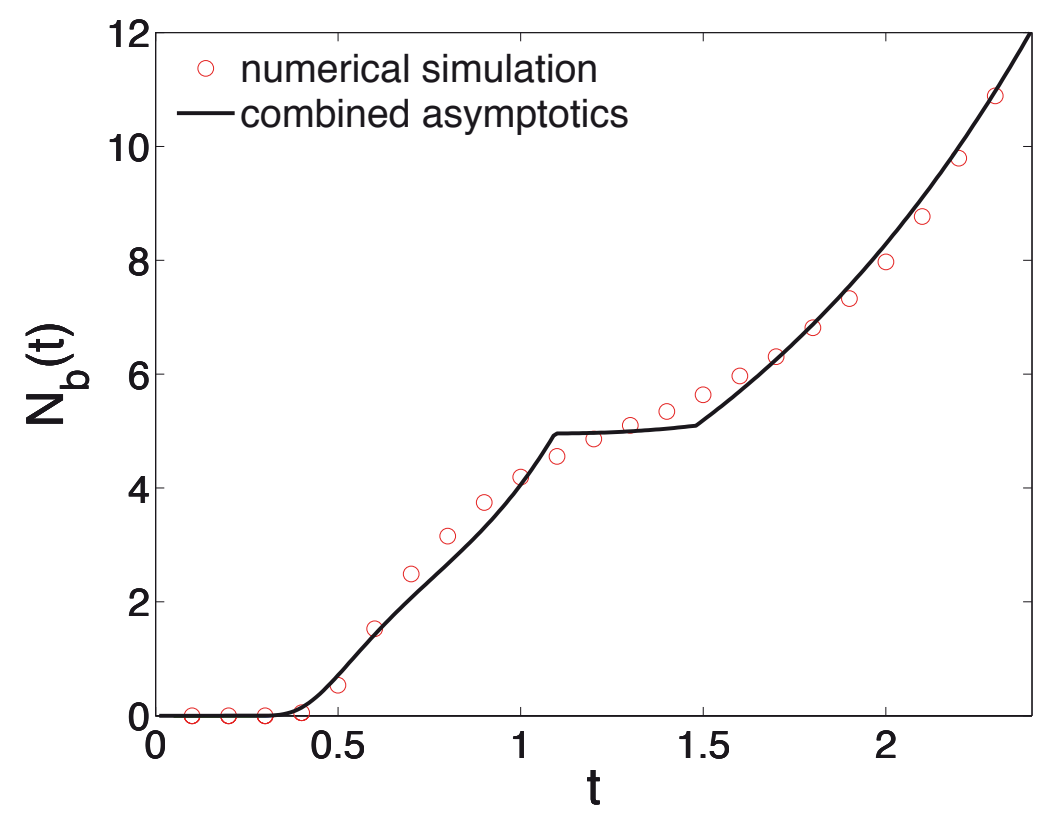




\section{Universal branch statistics}
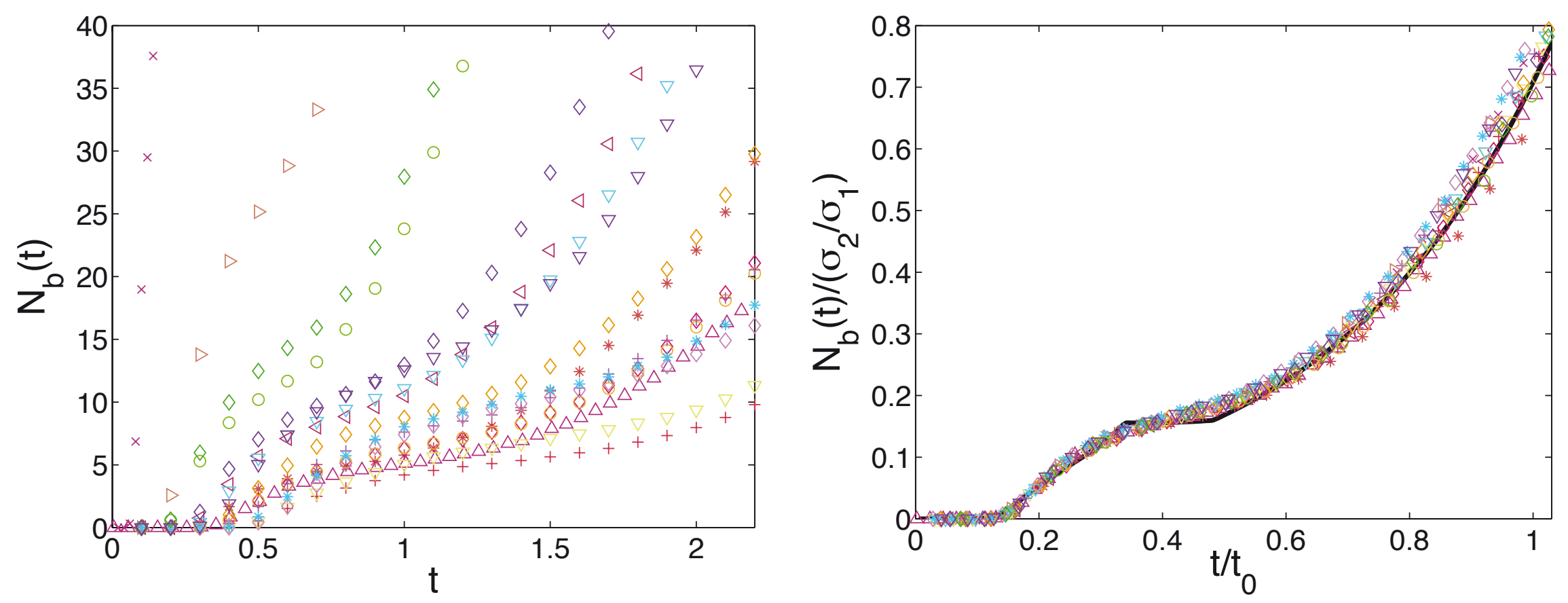

$\epsilon(\%) \ell_{c} c(r)$

$\epsilon(\%) \ell_{c} c(r) \quad \epsilon(\%) \ell_{c} c(r)$

$\begin{array}{rrr}+ & 4 & 0.1\end{array}$

* 80.1

\begin{tabular}{lll}
4 & 0.08 \\
\hline & 0.06
\end{tabular}

$\begin{array}{lll}4 & 0.06 \quad \mid\end{array}$

20.01

40.08 II

$\begin{array}{llll}6 & 0.08 & \text { II }\end{array}$

$\begin{array}{llll}4 & 0.06 \quad \text { II }\end{array}$

20.01 II

$\begin{array}{lll}4 & 0.1 & \text { III } \\ 2 & 0.08 & \text { III }\end{array}$

$\begin{array}{llll}* & 2 & 0.1 & \mathrm{IV}\end{array}$

( $)=0.1 \quad V$

$\begin{array}{llll}0 & 6 & 0.1 & V\end{array}$

$\begin{array}{lll}0.1 & \mathrm{I}(2 \mathrm{D})\end{array}$

$\begin{array}{llll}\nabla & 2 & 0.1 & \mathrm{VI}\end{array}$

$\diamond \begin{array}{lll}\diamond & 0.12 \mathrm{VI}\end{array}$

$$
\begin{aligned}
& c(r)=\epsilon^{2} \exp \left(-r^{2} / \ell_{c}^{2}\right) \\
& c(r)=\epsilon^{2} \operatorname{sech}\left(r / \ell_{c}\right) \\
& c(r)=\epsilon^{2}\left(1+r^{2} / \ell_{c}^{2}\right)^{-\alpha}
\end{aligned}
$$

(III) $-(\mathrm{VI})(\alpha=1,2,3,4)$ 


\section{Conclusions}

- Branched flow is a widespread phenomenon of flows in weak, correlated random potentials on scales smaller than mean free path

- Branch statistics can now be calculated for all distances from source and for a wide range of correlation functions
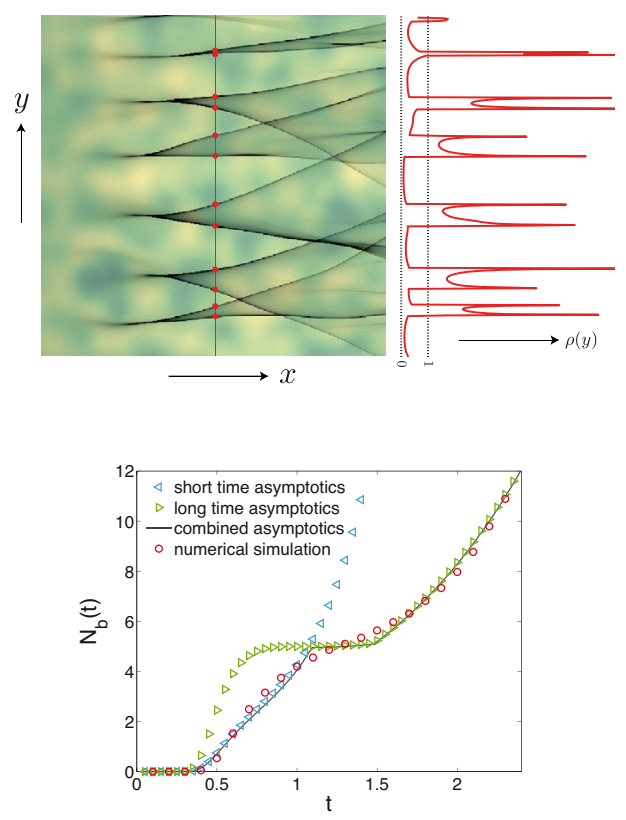

- Universal scaling of branch statistics
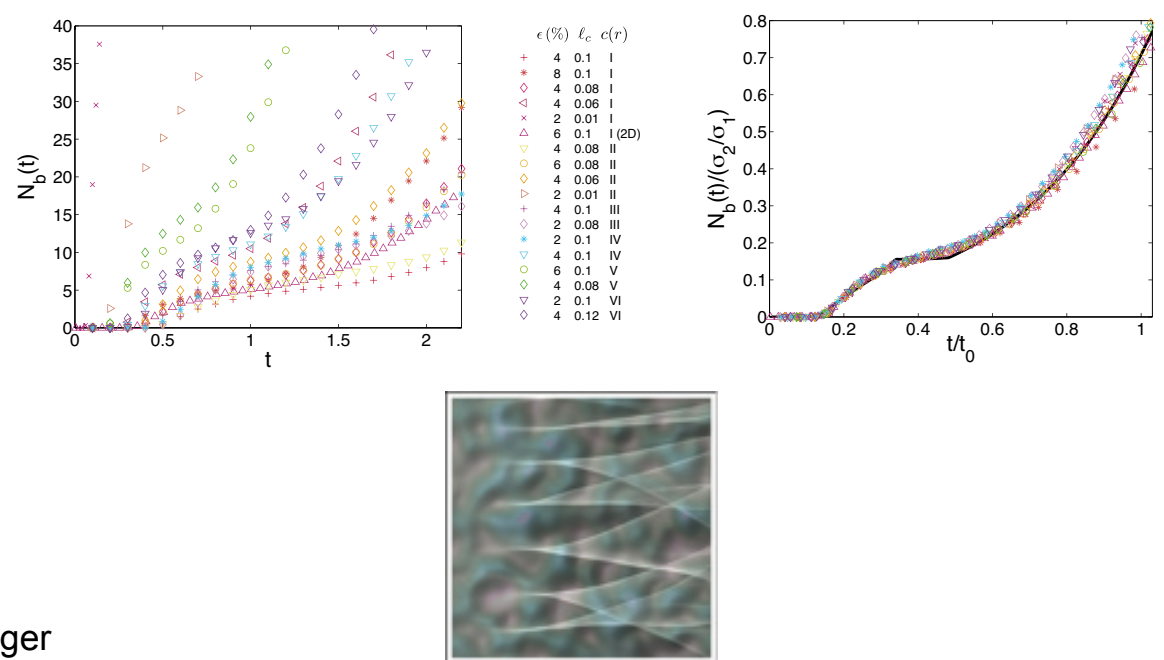